Service social

\title{
La violence psychologique envers l'enfant : quelques pistes d'action préventive à retenir
}

\section{Claire Chamberland et Andrée Fortin}

Volume 44, numéro 2, 1995

Visages de la violence

URI : https://id.erudit.org/iderudit/706696ar

DOI : https://doi.org/10.7202/706696ar

Aller au sommaire du numéro

Éditeur(s)

École de service social de l'Université Laval

ISSN

1708-1734 (numérique)

Découvrir la revue

Citer cet article

Chamberland, C. \& Fortin, A. (1995). La violence psychologique envers l'enfant : quelques pistes d'action préventive à retenir. Service social, 44(2), 129-146. https://doi.org/10.7202/706696ar
Résumé de l'article

La violence psychologique à l'endroit de l'enfant représente une des formes de mauvais traitements psychologiques. Elle est plus répandue que la violence physique et se manifeste dans toutes les classes sociales. Les conséquences sont nombreuses et souvent destructrices. Cet article tente de mettre en évidence différentes pistes d'intervention préventive susceptibles d'agir sur les déterminants à l'origine de ce phénomène. Les stratégies énoncées sont illustrées par des exemples de moyens et de programmes jugés prometteurs. L'efficacité relative de ces différentes stratégies doit être évaluée selon la nature et l'intensité des facteurs de risque et de protection en présence. 


\title{
La violence psychologique envers l'enfant: quelques pistes d'action préventive à retenir
}

\author{
Claire CHAMBERLAND* \\ École de service social \\ Université de Montréal \\ Andrée FORTIN* \\ École de psychologie \\ Université de Montréal
}

Le Groupe de travail pour les jeunes (Gouvernement du Québec, 1991) rappelait l'existence de milliers d'enfants victimes silencieusement des agressions verbales de leurs parents. Les résultats de la seconde enquête américaine sur la violence familiale menée auprès de 3346 familles indiquaient également un usage très répandu de la violence psychologique (Vissing, Straus, Gelles et Harrop, 1991). Près de $65 \%$ des enfants avaient subi au moins une fois I'agression verbale ou symbolique (insultes, injures, menaces, refus de parler) de leurs parents durant les 12 mois précédant l'enquête, le nombre moyen d'agressions étant de 12,6. Des résultats tout aussi alarmants ont été

\footnotetext{
* Les deux auteures sont membres du Centre de recherche interdisciplinaire sur la violence familiale et la violence faite aux femmes. Claire Chamberland est aussi directrice de l'Institut de recherche pour le développement social des jeunes.
} 
obtenus par Bouchard et Tessier (1996) auprès de familles d'un territoire défavorisé de la ville de Québec. Dans cet échantillon, 84,4\% des pères et $90 \%$ des mères reconnaissaient la présence de la violence psychologique dans la vie d'un enfant de la famille, ces taux étant supérieurs à ceux rapportés pour la violence physique mineure (les taux variant ici de $74 \%$ à $81,1 \%$ ). Certaines études rétrospectives suggèrent même que la proportion d'enfants victimes de la violence verbale de leurs parents atteindrait $90 \%$ (Briere et Runtz, 1988).

La violence psychologique ne renvoie pas à une réalité unique. Malgré les diverses appellations, on retrouve notamment un ensemble de conduites comprenant les expressions de rejet, de terrorisme, d'humiliation, de corruption ou d'isolement de l'enfant (Garbarino, Guttman et Seely, 1986). Malgré des difficultés importantes à définir et de surcroît à mesurer ce concept (Bouchard et Tessier, 1995), un consensus semble émerger voulant que la violence psychologique renvoie à un patron inapproprié de communication de l'adulte face à l'enfant. Ce patron pourrait mettre en péril la capacité de ce dernier à résoudre les tâches normales exigées par son développement (McGee et Wolfe, 1991), en tenant compte toutefois des normes sociales qui circonscrivent le caractère approprié ou inapproprié des conduites parentales (Manley et Cichetti, 1991). Les mauvais traitements psychologiques incluent à la fois les actes commis (abus) et les actes omis (négligence) jugés selon les standards de la communauté et l'expertise professionnelle comme étant psychologiquement dommageables (Hart et Brassard, 1982). Toutefois, la violence psychologique renvoie spécifiquement aux actes commis et par conséquent représente une des formes de mauvais traitements psychologiques (Malo, 1995).

Les conséquences de la violence psychologique que subit l'enfant sont nombreuses et apparaissent des plus destructives (Starr et Wolfe, 1990 ; Crittenden, Clausen et Sugarman, 1994). Chez les enfants victimes de violence verbale et émotive, la difficulté à former des relations interpersonnelles satisfaisantes, la culpabilité, la détresse et des comportements autodestructeurs sont au nombre des symptômes observés (Ney, 1987). Dans une synthèse de la documentation sur l'impact des mauvais traitements psychologiques, Hart, Germain et Brassard (1987) rapportent la présence de problèmes d'ordre physique (perte d'appétit, encoprésie, énurésie), affectif (dépendance, dépression, retrait), cognitif (incompétence, retard dans le développement intellectuel) et comportemental (vol, agressivité, prostitution); dans certains cas, les mauvais traitements conduiraient au suicide. 
Au fur et à mesure que les études lèvent le voile sur l'ampleur du phénomène de violence psychologique, s'accentue l'urgence de se doter d'outils de prévention efficaces, afin d'intervenir bien avant I'apparition de conduites parentales psychologiquement menaçantes et délétères pour le développement de l'enfant. Le texte qui suit dresse un bilan des interventions jugées prometteuses qui peuvent contribuer à diminuer ce problème psychosocial.

\section{LE MODÈLE ÉCOLOGIQUE: UN OUTIL POUR PRÉDIRE ET PRÉVENIR LA VIOLENCE PSYCHOLOGIQUE}

Dans une démarche de prévention qu'on veut efficace, on s'entend généralement pour cibler les interventions à deux niveaux: diminuer les facteurs de risque (facteurs qui augmentent la probabilité d'apparition des conduites violentes) et augmenter les facteurs de protection (facteurs qui neutralisent les effets de ces facteurs de risque). En promotion, le champ d'intervention s'inscrit d'emblée dans le cadre d'une dynamique positive. On renvoie à des déterminants et à des conditions favorables au développement (facteurs d'opportunité) plutôt qu'à des problèmes à éviter ou à résoudre (Chamberland, Dallaire, Cameron, Fréchette, Hébert et Lindsay, 1993). Le modèle écologique fournit un apport important non seulement à la compréhension de la problématique de la violence psychologique (Garbarino, Guttman et Seely, 1986; Hart, Germain et Brassard, 1987), mais aussi à la conception de programmes de prévention plus diversifiés (National Research Council, 1993; Olds et Kitzman, 1990). Adoptant une approche multifactorielle et multidimensionnelle, ce modèle postule que les comportements parentaux, abusifs ou non, résultent d'une interrelation complexe de facteurs positifs et négatifs situés à différents niveaux systémiques incluant l'enfant, sa famille et les contextes immédiats et plus éloignés qui les entourent (Belsky, 1980; Bronfenbrenner, 1979; Garbarino, 1982). Pransky (1991) a regroupé ces facteurs en une équation qui établit la probabilité de la violence faite à l'enfant en fonction du rapport qui existe entre les facteurs de risque (situés au numérateur) et les facteurs de protection (situés au dénominateur). Les facteurs de risque se regroupent de la façon suivante: les influences et pressions culturelles, le manque de possibilités et le stress de l'environnement ainsi que les dysfonctions familiales. Quant aux facteurs de protection, ils se divisent ainsi: saine perception de soi, compétences, niveau de conscience et soutien social. 
Cette conceptualisation d'un agenda en prévention repose sur I'importance accordée à l'action sur des déterminants génériques à l'origine non seulement du problème de violence psychologique à l'endroit des enfants, mais aussi des autres problèmes vécus par les jeunes. À cet effet, Pransky (1991) attire l'attention sur les liens existant entre les facteurs associés aux problématiques de mauvais traitement, de délinquance, de toxicomanie ou encore de violence conjugale. Par conséquent, prévenir la violence psychologique implique une action sur des déterminants multiples susceptibles de réduire également la probabilité d'apparition d'autres problèmes affectant le jeune.

\section{Les stratégies d'action}

Les pistes d'intervention décrites ici sont susceptibles d'affecter les différents éléments de l'équation de Pransky (1991). Pour chacune des stratégies énoncées, nous définirons les principaux objectifs à poursuivre, de même que les méthodes d'action qui y sont les plus pertinemment associées. Puis, afin d'illustrer plus concrètement ces mêmes stratégies, et par la même occasion inspirer plus directement le développement de pratiques préventives ou promotionnelles, nous présenterons des exemples d'actions ou de programmes prometteurs et jugés efficaces.

\section{Modifier les attentes culturelles}

Le premier élément de l'équation de Pransky (1991) fait référence aux attentes culturelles. On pense ici à neutraliser les systèmes de croyance, les valeurs et les attitudes qui cautionnent et banalisent le recours à la violence ainsi qu'à des conduites de contrôle de l'enfant qui sont exagérément coercitives ou encore qui définissent l'enfant comme la propriété du parent. À ce niveau, trois objectifs doivent être poursuivis. Le premier vise à mettre en évidence les différentes formes de mauvais traitements, en particulier ses manifestations les plus subtiles, voire les plus invisibles. Le deuxième consiste, d'une part, à dénoncer l'impact négatif de la télévision sur l'acquisition de normes et de comportements violents et, d'autre part, à mieux exploiter son apport positif, faisant ainsi en sorte que ce puissant médium ne fasse pas partie du problème mais plutôt de sa solution (Gouvernement du Québec, 1991). Enfin, le troisième consiste à promouvoir une idéologie pro-enfant qui valorise l'enfant comme une personne à part entière (Hart, 1988; Melston, 1988). 
Concrètement, les méthodes $d^{\prime}$ intervention en cause relèvent de l'éducation en petit groupe ou auprès de l'ensemble de la population. Dans le dernier cas, les moyens de communication de masse ou de marketing social constituent des outils privilégiés pour dénoncer les conduites et attitudes psychologiquement maltraitantes ou, plus positivement, pour influencer l'adoption de valeurs plus souhaitables au sein de l'ensemble de la population (Perreault, 1995). Ainsi les campagnes de sensibilisation visant le grand public et insistant sur le fait que les mots peuvent "tuer» représentent des efforts louables susceptibles de générer de nouveaux schémas d'interprétation des conduites parentales. Au Québec, le ministère de la Santé et des Services sociaux a produit une publicité qui illustre à quel point les enfants sont importants dans nos vies et l'ampleur de I'influence des adultes sur ceux-ci. Une initiative plus récente diffusée sur l'ensemble des réseaux d'État et illustrant des formes de violence interpersonnelle trop souvent ignorées constitue une autre intervention de même type que l'on pourrait très bien appliquer aux relations parent-enfant.

Les émissions éducatives comme les téléromans, les magazines d'information ou les vidéos mettant en scène des situations familiales problématiques contribuent ainsi à mieux nommer et à reconnaître des pratiques éducatives répréhensibles. Ciblant les jeunes, les parents ou l'ensemble de la famille, de telles productions peuvent constituer une excellente occasion d'interactions et de partage de points de vue à propos de modèles de comportements et de valeurs. Ces outils sont d'autant plus puissants que la télévision représente pour l'enfant une fenêtre sur le monde (Eron et Huesmann, 1987)'.

Enfin, il ne faudrait surtout pas passer sous silence l'importante contribution de moyens sociopolitiques dans la promotion des droits des enfants. En effet, la Convention des Nations-Unies sur les droits de l'enfant adoptée par l'ONU en 1989 constitue un véritable traité qui créera des obligations précises à l'égard des États qui I'auront ratifiée. Elle propose une véritable révolution des valeurs et des mentalités en ce qu'elle témoigne d'une nouvelle vision universelle de l'enfant, vu non pas comme un objet, mais comme un sujet de droits, une personne à part entière (Joyal, 1990). Toutefois pour que cette convention généreuse ne demeure pas lettre morte, une mobilisation des personnes et des groupes préoccupés de justice sociale et de solidarité est requise.

1. Incidemment, les auteures du présent article travaillent actuellement à la conception d'une série télévisuelle qui ciblerait l'ensemble de la famille, en concertation avec une chaîne de télévision québécoise; cette production a pour objectif principal de prévenir non seulement la violence physique mais aussi psychologique à l'intérieur de la famille (Fortin, Chamberland, Gascon et Carignan, 1995). 


\section{Les stress socio-environnementaux et le manque d'opportunité}

Les sociétés occidentales ont contribué à susciter des processus et des structures pour diminuer l'écart de pouvoir entre les individus et assurer les bien-être économique, social et symbolique des familles. Ces trois types de bien-être nécessitent I'accès à des conditions de vie qui assurent respectivement les besoins matériels des familles (logement, revenu/emploi, alimentation, vêtements), leurs besoins $d^{\prime}$ action, d'intégration et d'influence au sein des réseaux primaire et secondaire (rôles sociaux et communautaires) ainsi que leurs besoins de reconnaissance et de fierté liés à la formation d'une identité positive, personnelle et sociale (Pransky, 1991; Chamberland, 1995).

Mais force est de constater qu'entre les idéaux et la réalité un fossé considérable reste à franchir. Les menaces au bien-être de la famille sont encore nombreuses et constituent des facteurs de risque de violence psychologique envers les enfants. Les stress reliés à la pauvreté ne sont pas les seuls en cause. La poursuite incessante de I'excellence, du prestige, de privilèges et de biens représente une source de stress trop souvent négligée et pourtant fortement associée à la violence psychologique (Garbarino, 1986). Du reste, cette situation risque d'être exacerbée dans le contexte actuel de décroissance et de restructuration économique où bon nombre de parents n'ont plus leur place ou encore doivent sacrifier leur vie familiale pour la conserver. La lutte des places est aussi impitoyable que la lutte des classes (De Gaujelac et Taboada Leonetti, 1994).

L'action centrée sur les stress avec lesquels les familles sont aux prises demeure le parent pauvre de la prévention. En général, les efforts sont axés sur le «stress management» plutôt que sur l'éradication des stresseurs et des structures sociales qui les produisent. Dans une étude récente menée au Québec, environ $2 \%$ des projets recensés en matière de prévention-promotion dans le domaine Enfance/Famille (sur un total de 302 projets implantés dans 25 territoires de CLSC différents) rapportent déployer des stratégies collectives pour améliorer ou revendiquer de meilleures conditions de vie ou des rapports sociaux plus égalitaires (Chamberland et Dallaire, 1994; Chamberland, Fréchette, Hébert et Lindsay, 1994). Néanmoins, $18 \%$ des projets mentionnent apporter un soutien concret destiné à soulager, sur une base individuelle, les familles ciblées (endettement, logement, nourriture, répit, etc.). L'initiative de ces actions est presque entièrement soutenue par les milieux communautaires. La majorité des interventions visent le renforcement du potentiel des personnes ou le soutien social des familles et des jeunes. Ces observations 
corroborent celles déjà constatées dans la recension de Malo (1991) portant sur un inventaire de programmes de prévention américain et canadien jugés les plus prometteurs.

L'intervention multisectorielle $s$ 'avère indispensable pour rendre cohérentes l'ensemble des actions visant la diminution des stress socio-environnementaux. Au Québec, quelques exemples méritent d'être soulignés : 1) la création du Conseil de la famille ainsi que d'un Conseil permanent de la jeunesse qui veille aux intérêts de cette partie de la population; 2) des événements susceptibles de mobiliser I'attention et l'énergie autour de certains enjeux économiques, notamment le Forum sur l'emploi et un colloque sur la fiscalité des familles; 3) plusieurs regroupements qui revendiquent des logements sociaux, une sécurité de revenu ou encore qui défendent et conseillent les familles dans leur rapport à la consommation ou dans leur relation parfois conflictuelle avec différentes institutions, notamment I'aide sociale, le milieu de travail, l'école; 4) et même un outil récemment conçu pour faciliter la mise en place de stratégies de concertation sur une base locale. Cet outil propose une démarche facilitant la coordination des actions d'un ensemble de ressources qui possèdent des mandats et des fonctionnements différents, afin d'obtenir une plus grande cohérence entre divers projets qui ont recours à plusieurs stratégies en vue de modifier plus d'un déterminant (Ouellet et Paiement, 1993). Cinq étapes pas nécessairement envisagées selon un ordre linéaire, mais dont la logique de développement suit plutôt un mouvement circulaire et de va et vient, sont suggérées : 1) la mobilisation des partenaires ; 2) l'établissement d'un consensus de base ; 3) la mise en commun des projets et des besoins (analyse du milieu);

4) I'élaboration d'un plan d'action et enfin 5) I'implantation et l'évaluation.

En somme, pour réduire les stress socio-environnementaux et augmenter les possibilités, il faut améliorer le pouvoir économique, politique et civique des familles et des jeunes et ainsi réduire I'exclusion et la marginalisation. En d'autres termes, il faut contrer la violence institutionnelle et sociale, laquelle mine la vie et le climat des familles. Enfin, la violence psychologique étant un problème multidéterminé, l'action intersectorielle devient une condition nécessaire pour espérer enrayer efficacement ce phénomène.

\section{Les déficits dans les familles}

En prévention primaire, I'intervention survient avant l'apparition du problème (Lafortune et Keiley, 1989). Néanmoins, certains parents peuvent éprouver des difficultés personnelles, relationnelles, 
situationnelles ou économiques qui entravent sérieusement leur capacité à prendre soin d'un enfant, de même qu'à faire face aux nombreux défis que pose son éducation. Dans ces cas, on cible davantage les familles dites à risque et pour lesquelles on doit parfois réparer les effets d'un passé douloureux ou "corriger » le plus précocement possible des interactions, des conduites ou des attitudes dont I'impact est répréhensible pour le développement de l'enfant. Lorsque des signes avant-coureurs indiquant la mise en place d'un patron de communication psychologiquement abusif sont déjà observés entre le parent et l'enfant, la prévention est toutefois de nature secondaire (Chamberland, 1992). Mentionnons dès le départ que lorsque le parent est ciblé, cela comprend aussi bien le père que la mère. En effet, le père est souvent le grand absent ou à tout le moins le parent le plus négligé dans les agendas en prévention-promotion. Comme le soulignait très justement le groupe de travail sur les jeunes (Gouvernement du Québec, 1991), il est important de convaincre le père de la nécessité de sa présence et de son rôle auprès de l'enfant et ce, dès sa naissance. Il s'agit de prévenir le décrochage du père ou ce que certains appellent le désengagement paternel (Quéniart et Fournier, 1995). Au moment d'une séparation ou d'un divorce, environ $50 \%$ des pères non gardiens perdraient à peu près tout contact avec leur enfant. C'est pourquoi, même si les projets de prévention présentés subséquemment ont souvent plus ciblé les mères que les pères, ils nous apparaissent également pertinents pour les deux parents.

Lorsque le fonctionnement familial est problématique, les objectifs importants à poursuivre sont multiples et consistent, notamment, à modifier les attentes irréalistes, à pallier le manque de préparation du parent (Erickson et Egeland, 1987), à satisfaire les besoins psychologiques et sociaux de base de parents qui ont vécu des enfances difficiles (Main et Goldwyn, 1984) et à éliminer les patterns de fonctionnement problématiques au sein de la famille (Glaser et al., 1993). Qu'ils soient orientés vers le parent, le couple ou l'ensemble de la famille, les moyens d'intervention sont souvent de nature plus clinique. La démarcation entre le préventif et le curatif dans ces cas est plus ambiguë. Moins l'enfant aura subi d'effets attribuables aux conduites des parents, plus l'intervention sera considérée comme étant de nature préventive. Ainsi, agir durant la grossesse ou durant les premières années de vie de l'enfant assure le caractère plus proactif de l'intervention.

Parmi les initiatives à retenir, notons l'approche de Fraiberg (1980). La clé de ce type d'intervention réside dans le développement progressif $d^{\prime}$ une relation de confiance qui s'établit entre une éducatrice et la mère en difficulté. C'est une véritable thérapie de cuisine 
où l'intervenante écoute de manière non blâmante et non critique le parent raconter I'histoire parfois douloureuse de sa vie. À I'aide de cette relation de compassion et de confiance, la mère apprend à décoder les besoins de son propre enfant et à acquérir un sentiment de protection et de sollicitude. Dans la foulée de cette expérience affective, la mère acquiert une meilleure image d'elle-même et se rapproche de son enfant, découvrant sa capacité de le consoler, de le protéger et de l'amener à vivre ce dont elle a souvent cruellement manqué. C'est à partir du lien d'attachement mère-enfant que se constitue la barrière de protection fondamentale contre la maltraitance (Crittenden, 1988; Gauthier, 1992). Le plus grand défi réside dans le dosage entre les aspects éducatif et psychothérapeutique de l'intervention.

Une perspective plus systémique est également recommandée pour assainir les patterns d'interaction du couple et de l'ensemble de la famille. Notons par exemple l'amélioration des capacités à résoudre des conflits (Wolfe, 1985), à inhiber les sentiments de colère et de frustration par différentes méthodes de «time out » (Veenstra et Scott, 1993) ou encore la promotion d'une qualité affective dans les relations familiales (Emery, 1989). Enfin, puisque ces familles sont régulièrement confrontées à une panoplie de difficultés, un des enjeux principaux consiste à harmoniser les différentes stratégies mises en place (I'emploi, le logement, les troubles d'adaptation personnelle et sociale, l'isolement social, la santé, le manque d'autorité parentale). Il en va de l'efficacité de l'intervention (Schorr, 1988).

\section{Le développement de compétences, la conscientisation et la perception de soi positive}

Lorsqu'on contribue à ajouter ou à consolider des forces chez une personne, on s'éloigne des modèles psychopathologiques davantage centrés sur les déficits. Mettre en place des conditions favorables au bien-être des enfants et des familles s'inscrit dans la tradition des approches en promotion (Blanchet et al., 1993). Par analogie, un fumeur doit non seulement éliminer l'habitude néfaste de fumer pour aspirer à améliorer sa santé et son bien-être, mais aussi apprendre à faire de l'exercice, mieux s'alimenter ou avoir des loisirs stimulants. Au regard de la prévention de la violence psychologique, les cibles visées peuvent être les enfants, les parents ou les deux à la fois. Il faut aussi inclure les autres agents de socialisation normalement engagés dans l'éducation des enfants, comme les professeurs (Strickland et 
Campbell, 1982 ; Erickson et Egeland, 1987) ou encore les intervenants en milieu de garde et dans la communauté (notamment dans les milieux de loisirs).

Plus généralement les objectifs visent à répondre aux besoins de base des personnes: avoir un sentiment d'appartenance, donner et recevoir de l'amour, avoir le sens de sa propre valeur et enfin avoir du pouvoir, de la liberté et, bien sûr, du plaisir (Glasser et Powers, 1981). D'autres objectifs sont plus directement orientés vers I'acquisition d'attitudes et de compétences du parent, de l'enfant et consistent aussi à favoriser des liens d'attachement entre eux (Hart, 1988). Il s'agit ici de créer des contextes où le parent et le jeune peuvent acquérir un concept de soi positif, I'autodiscipline, l'autocontrôle, des habiletés sociales, notamment l'art de communiquer et la résolution de problèmes interpersonnels ainsi que des compétences à gérer efficacement les stress. Enfin, il importe également de planifier des situations où parents et enfants auront la possibilité $\mathrm{d}^{\prime}$ actualiser leurs potentiels et leurs talents ainsi que d'avoir une rétroaction claire de leur pouvoir personnel. En général, ces types d'initiatives représentent une proportion importante des efforts en prévention. Dans la recension de Malo (1991), près de $75 \%$ des projets ciblant les enfants, les adolescents et les parents sont articulés en termes de développement des compétences. C'est le cas de $32 \%$ des 302 projets étudiés dans une étude québécoise (Chamberland et al., 1994). En règle générale, l'intervention se déroule dans le cadre de visites à domicile, de groupes d'éducation ou encore à l'hôpital après l'accouchement. Lorsque le programme s'adresse uniquement aux jeunes, la priorité est souvent accordée au milieu scolaire. Les programmes qui s'adressent aux parents ou à l'ensemble de la famille se concentrent surtout autour des compétences parentales. Contrairement aux projets ou activités portant sur des déterminants plus macro-exosystémiques qui, par leur nature, sont plus complexes à évaluer, l'ensemble des projets mentionnés dans cette section (et la suivante) ont bénéficié d'évaluation systématique. Ils ont été sélectionnés précisément pour leur capacité à mettre en évidence des gains statistiquement significatifs.

Parmi les programmes retenus, certains s'adressent aux parents (incluant la relation parent-enfant), alors que d'autres ciblent surtout les jeunes. Dans le premier cas, quatre programmes sont à retenir parce qu'ils ont démontré une approche pertinente et laissent entrevoir des résultats prometteurs. L' «Optimum Growth Project», réalisé en Floride (Dusenbury-Kelly, 1982, cité dans Malo, 1991), a reçu en 1980 le Lela Rowland Prevention Award. L'intervention débute dès le 
troisième mois de grossesse et se termine lorsque l'enfant atteint l'âge de six ans. Une intervenante non professionnelle rend visite à la mère et lui apprend à stimuler son enfant, la conseille et lui fournit des informations et des références. L'évaluation rapporte une plus faible fréquence de mauvais traitements et moins de grossesses rapprochées. Le «Milwaukee Project» (Heber et Garber, 1975 dans Malo, 1991) adopte une approche semblable et inclut également des groupes de stimulation pour les enfants ainsi qu'une incitation pour les mères à s'insérer dans le marché du travail. L'étude d'impact révèle des acquisitions particulièrement pertinentes eu égard à la prévention de la violence psychologique. Notamment, les mères utilisent plus de renforcements verbaux avec l'enfant et les enfants sont verbalement plus expressifs. Le "Houston Parent Child Development Center» (Johnson et Breckenridge, 1982, cité dans Malo, 1991) est I'un des modèles primés par l'American Psychology Association (APA). Le plan d'intervention comporte aussi des visites à domicile effectuées par une mère expérimentée qui fournit informations et techniques de soins. Des ateliers de fin de semaine sont également organisés et portent sur l'acquisition d'habiletés de communication et de prise de décision. À cela s'ajoutent des groupes éducatifs abordant des questions comme la sécurité au travail et le budget, tandis que les enfants bénéficient de séances d'entraînement à différentes compétences. L'évaluation met en évidence des gains significatifs tant pour les mères que pour les enfants: celles-ci seraient plus affectueuses, formuleraient moins de critiques, seraient moins rigides, les encourageraient davantage et leur fourniraient un environnement plus stimulant. Les enfants seraient plus sensibles émotivement et auraient moins de comportements destructeurs. Le "Stress Management Training » (Tableman, 1987, cité dans Malo, 1991), reconnu en 1985 par le Lela Rowland Prevention Award, favorise I'acquisition, par des femmes à faibles revenus, de compétences à planifier leur vie, à prendre des décisions, à résoudre des problèmes et à gérer des stress. L'évaluation indique notamment une meilleure capacité chez ces femmes à contrôler leur vie, à reconnaître leurs émotions et à améliorer leur estime d'elles-mêmes. Nous pouvons raisonnablement présumer que ces changements sont susceptibles d'affecter positivement leur relation avec leur enfant. II serait d'ailleurs souhaitable que des programmes analogues visent de manière spécifique les pères, ces grands absents de l'intervention psychosociale en général.

Les programmes s'adressant aux enfants s'avèrent pertinents pour deux raisons: d'une part, ils permettent de développer des facteurs de protection pour neutraliser l'effet de conditions familiales 
adverses et, d'autre part, ils outillent plus adéquatement l'enfant comme futur parent. Trois programmes nous apparaissent particulièrement intéressants. D'abord le High/Scope Perry Preschool Program (Berrueta-Clement et al., 1984, cité dans Malo, 1991) représente I'un des 14 modèles d'intervention proposés par I'APA. Les enfants participent pendant deux ans à des groupes de stimulation et les parents sont également vus à leur domicile. Le suivi longitudinal de cohortes d'enfants a permis de relever des effets notables du programme dans le cadre de la prévention de la violence psychologique. Devenus jeunes adultes, les participants ont des probabilités moindres de vivre des stress négatifs telles la grossesse précoce, une sous-scolarisation et une dépendance aux prestations de l'État. Les deux autres programmes visent à développer chez l'enfant des habiletés à résoudre des problèmes (Shure et Spivack, 1982, cité dans Malo, 1991) ainsi que des compétences à s'affirmer (Rotheram et al., 1982, cité dans Malo, 1991). L'évaluation démontre des gains substantiels sur le plan des compétences à trouver des solutions de rechange, à identifier les conséquences, à déployer des compétences non verbales et à contrôler les émotions.

\section{Le soutien social}

Les approches d'entraide et d'aide par les pairs ou aidants naturels représentent des méthodes d'intervention très courantes dans le champ de la prévention. Depuis quelques années, plusieurs types d'intervention axés sur la mobilisation du soutien social ou la création de réseaux de soutien informel ont proliféré (Tousignant et al., 1987). Ils constituent une solution de remplacement pertinente du réseau formel. Parmi les avantages de ce système d'aide, notons I'accessibilité, la congruence avec les normes locales, I'enracinement dans des relations à plus long terme, la diversité des stratégies d'aide ou encore le caractère réciproque des échanges qu'il favorise (Gottlieb, 1987 ; Rook et Dooley, 1985). Certains auteurs soulignent qu'il est préférable de créer des contextes de soutien aux parents plutôt que de constituer des groupes didactiques centrés sur l'éducation parentale (Child Abuse and Neglect, 1991). Les différents groupes de "Parents anonymes» (Dallaire et Chamberland, 1994) ou les approches de marrainage (Durand, Massé et Ouellet, 1989) centrées sur l'acquisition de compétences parentales par la mise en commun des expériences souvent très variées des parents illustrent ce type d'initiative.

Les interventions axées sur le soutien social représentent près de $50 \%$ des stratégies observées dans les 302 projets en Enfance- 
Jeunesse-Famille répertoriés dans une recherche récente au Québec (Chamberland et al., 1994). Deux grandes catégories d'objectifs sont généralement poursuivis. Le premier objectif consiste à mobiliser et à améliorer le soutien déjà existant, soit par l'intervention d'aidants naturels dans la communauté, l'entraide par les pairs, la stimulation des liens intergénérationnels ou la sensibilisation à l'importance d'utiliser son réseau d'aide. Quant au second, il vise surtout à augmenter le soutien aux parents ou aux jeunes, étant entendu que le soutien apporté par le réseau n'est pas suffisant pour fournir les ressources émotive, cognitive, normative ou instrumentale nécessaires. $L^{\prime}$ insertion dans des groupes d'entraide, les liens engendrés par des stratégies de marrainage ou de parrainage, la création de ressources de garde, de répit ou de loisir ainsi que les approches communautaires dans le quartier sont quelques-uns des moyens pertinents pour contrer l'isolement des familles et des jeunes.

Parmi le vaste choix de programmes agissant à ce niveau, trois programmes retiennent notre attention. Le premier projet a été largement cité dans les écrits comme un des modèles à mi-chemin entre I'éducation parentale et l'action sur le réseau d'aide. Le "Prenatal Early Infancy Project»(Olds et al., 1986), également primé par l'APA, est encore considéré comme I'un des programmes les plus prometteurs pour prévenir la maltraitance, et ce particulièrement auprès de jeunes mères aux prises avec d'importants stress économiques (MacMillan, MacMillan, Offord, Griffith et MacMillan, 1994). Il vise à favoriser l'accès à du soutien par la voie de visites à domicile effectuées par une infirmière qui, en plus de fournir de l'information à partir d'un contenu structuré mais flexible, aide la jeune mère à diminuer son stress, à utiliser les ressources communautaires ainsi qu'à inciter le réseau à s'impliquer auprès d'elle. En somme, ce projet vise l'intégration du soutien formel et informel de la communauté. L'évaluation est éloquente. Durant la grossesse, les mères ont davantage recours aux services et rapportent une présence plus importante de leur réseau d'aide. Leur bébé est, à la naissance, en meilleure santé et les signalements aux services de protection sont moins nombreux. Le «Neighborhood Parenting Support Project» (Lugtig et Fuchs, 1992) est un exemple intéressant de développement communautaire à l'intérieur d'un quartier où les taux de signalement pour mauvais traitements sont élevés. On cherche à y diversifier et élargir les sources d'aide de familles dites à risque, à favoriser I'appropriation des parents dans la communauté et à mieux harmoniser les réseaux d'aide informel et formel. L'impact sur le réseau d'aide des familles et l'entraide dans le quartier a été non négligeable. Ainsi, les parents qui présentaient des risques ont appris à avoir davantage 
recours aux amis et aux voisins plutôt que de compter exclusivement sur la parenté. En outre, un lieu d'échange pour les parents du quartier a été créé, un bottin énumérant l'ensemble des ressources du quartier a été conçu par deux parents et distribué dans mille foyers et, enfin, une coopérative de gardiennage a été mise sur pied.

Enfin, la campagne d'éducation publique auprès de l'ensemble de la population californienne intitulée "Friends can be a good medicine» (Taylor, Lain, Roppel et Barter, 1984, cité dans Malo, 1991) est un excellent exemple à mi-chemin entre l'action sur les attentes culturelles et la promotion du soutien social. Les objectifs consistent à informer le public du rôle que joue le soutien social sur la santé et le bien-être, à encourager l'investissement de temps et d'énergie dans les relations humaines et à renforcer les réseaux d'entraide et communautaires. Les stratégies utilisées portent essentiellement sur des moyens de communication de masse et de marketing social: messages à la radio et à la télévision, diffusion d'un film sur le thème des amis, brochure pour permettre d'auto-évaluer son propre système de soutien, affiches et activités communautaires. L'évaluation montre des changements d'attitudes et des intentions comportementales favorables à la promotion du soutien social.

\section{CONCLUSION}

Dans le présent article, nous avons rapporté plusieurs stratégies déjà disponibles pour prévenir la violence psychologique envers l'enfant. Ces stratégies s'inspirent des modèles d'action propres aux communications de masse et au champ de l'intervention éducative ou encore s'associent aux interventions de nature clinique, communautaire, socio-économique et politique. Pour réduire de manière sensible les mauvais traitements psychologiques au sein de la population, nos efforts doivent être non seulement soutenus, mais augmentés. Aussi faut-il lutter contre les idéologies qui favorisent les interventions de crise et les approches curatives au détriment des interventions préventives (Hart, 1988). Cela est d'autant plus essentiel dans un contexte socio-économique difficile où la rareté des ressources favorise l'augmentation du seuil de tolérance aux problèmes sociaux plutôt que la mise en place d'interventions pertinentes (Giovannoni, 1991).

Pour améliorer l'efficacité des interventions et stimuler le développement de nouvelles stratégies, des questions importantes restent à élucider. Ainsi il est possible que la présence simultanée de certains facteurs de risque soit plus déterminante dans la vulnérabilité à la 
violence psychologique. Il se peut également que le développement de certains facteurs de protection soit davantage pertinent eu égard à la présence de facteurs de risque particuliers.

Dans certains cas, des interventions axées uniquement sur I'acquisition de compétences parentales ne sont peut-être pas à favoriser lorsque la famille est soumise à des stress socio-environnementaux intenses. Renforcer les capacités personnelles et suréquiper le parent, alors que l'environnement est truffé de risques, peut se révéler une solution décevante, voire stérile (Bouchard, 1988). L'insertion dans des réseaux communautaires apparaît peut-être un moyen plus pertinent pour contrer l'effet combiné et explosif de la pauvreté et de l'isolement. Cette dernière stratégie a de plus l'avantage de diversifier les types de soutien pour appuyer le parent dans son rôle auprès de l'enfant. Dans d'autres cas, l'addition de stratégies $d^{\prime}$ acquisition de compétences et $d^{\prime}$ insertion dans des groupes d'entraide peut s'avérer une solution prometteuse, alors que chacune des deux méthodes appliquées isolément limite la portée de l'intervention (Dallaire et Chamberland, 1994).

En somme, l'efficacité relative de différentes stratégies doit être évaluée selon la nature et l'intensité des facteurs de risque et de protection en présence. Seules des évaluations systématiques et rigoureuses portant sur la pertinence des composantes à intégrer à I'intérieur d'un programme permettront de fournir des solutions durables pour éliminer ce problème.

\section{Références bibliographiques}

BELSKY, J. (1980). "Child maltreatment: An ecological integration», American Psychologist, 35 : 320-335.

Blanchet, L., M.-C. Laurendeau, D. PAul et J.-F. SAuCier (1993). La prévention et la promotion en santé mentale: préparer l'avenir. Boucherville: Gaëtan Morin Éditeur.

BOUCHARD, C. (1988). La pauvreté comme une courbe dangereuse. Montréal: Institut Vanier de la famille.

BOUCHARD, C. et R. TESSIER (1996). "La violence familiale envers les enfants: validité de mesure et prévalence dans un quartier populaire urbain», dans R. Tessier, G.M. Tarabulsy et L.S. Éthier, Dimensions de la maltraitance. Sainte-Foy: Presses de l'Université du Québec.

BOUCHARD, C. et R. TESSIER (1995). "Conduites à caractère violent à l'endroit des enfants», dans C. Lavallée, M. Clarkson et L. Chénard (dir.), Conduites à caractère violent dans la résolution de conflit entre proches. Enquête sociale et de santé 1992-1993, Monographie $n^{\circ} 2$, p. 21-74. 
Montréal : Ministère de la Santé et des Services sociaux, Gouvernement du Québec.

BRONFENBRENNER, U. (1979). The ecology of human development: experiments by nature and by design. Cambridge, MA : Harvard.

CARUSO, G.A.L. (1989). "Optimum growth project: Support for families with young children », Prevention in Human Services, $n^{\circ} 6$ : 123-139.

CHAMBERLAND, C. (1995). Lutter et prévenir la pauvreté des enfants: une question d'éthique et de savoir-faire. Actes du colloque sur la pauvreté des enfants. Caledon Institute of Social Policy, p. 10 à 20.

CHAMBERLAND, C. (1992). "La violence faite aux enfants: la comprendre pour mieux la prévenir», Prisme, $3: 16-28$.

Chamberland, N., L. FréChetTe, J. HéBert et J. LindSAy (1994). «Prévention sociale; stratégies et critères d'efficacité », VI Congrès en prévention. Toronto.

Chamberland, N. et C. DALlaire (1994). Les cadres des analyses et des modèles d'action en prévention Enfance-Jeunesse-Famille. Communication présentée à l'École de psychologie de I'Université Laval, Québec.

Chamberland, N., N. Dallaire, S. Cameron, L. FréChette, J. Hébert et J. LINDSAY (1993). "La prévention des problèmes sociaux: réalité québécoise », Service social, vol. 42, n 3: 55-81.

CHILD ABUSE AND NEGLECT (1991). "Recommandations for the 21st century ", Child abuse and neglect, vol. 15, sup. 1:39-50.

CRITTENDEN, P.M. (1988). "Relationships at risk», dans J. Belsky et J. Nezworsky (dir.), Clinical Implications of Attachment (p. 23-44). Illsdale: Lawrence Erlbaum.

CRITTENDEN, P.M. (1988). "Family and dyadic patterns of functioning in maltreating families», dans K. Browne, C. Davies et P. Stratton (dir.), Early prediction of child abuse. New York: Wiley.

Crittenden, P.M., A.H. Claussen et D.B. Sugarman (1994). "Physical and psychological maltreatment in middle childhood and adolescence », Development and Psychopathology, 6 : 145-164.

DALLAIRE, N. et C. CHAMBERLAND (1994). "La pertinence du programme "Parents efficaces" auprès de parents bénéficiaires du soutien d'un groupe "Parentraide" : une étude préliminaire, Revue canadienne de service social, vol. 11, $\mathrm{n}^{\circ} 1: 67-88$.

De Gaujelac, V. et I. TABOAda LeOnetti (1994). La lutte des places. Paris : Epi.

DURAND, D., R. MASSÉ et F. OUELLET (1989). «Intervenantes non professionnelles et prévention de l'enfance maltraitée: évaluation du projet De la Visite», Santé mentale au Québec, vol. XIV, n 2 : 26-38.

DUSENBURY-KELLY, L. (1982). "Between the dream and the reality: A look at programs nominated for the Lela Rowland Prevention Award of the National Mental Health Association ». Journal of Primary Prevention, $2: 217-239$.

EMERY, R.E. (1989). "Family violence», American Psychologist, 44 : 321-328.

ERICKSON, M.F. et B. EGELAND (1987). "A developmental view of the psychological consequences of maltreatment », School Psychology Review, 16: 156-168. 
ERON, L.D. et L.R. HUESMANN (1987). "Television as a source of maltreatment of children », School Psychology Review, 16 : 195-222.

Fortin, P., C. Chamberland et A. CARIGNAN (1994). Projet de série télévisée sur la prévention de la violence familiale. Montréal: Université de Montréal, Centre de recherche interdisciplinaire sur la violence familiale et la violence faite aux femmes.

FRAIBERG, S. (1980). Clinical studies in infant mental health: The first year of life. New York: Basic Books.

GARBARINO, J. (1982). Children and families in the social environment. New York: Aldine Publishing Company.

Garbarino, J., E. GutTMAN et J. SEELEy (1986). The psychologically battered child: Strategies for identification, assessment and intervention. San Francisco : Jossey-Bass.

GAUTHIER, M.-C. (1992). "L'intervention auprès des jeunes familles en difficulté », Prisme, $3: 40-49$.

GIOVANNONI, J. (1991). "Social policy considerations in defining psychological maltreatment», Development and Psychopathology, 3 : 51-59.

Glasser, B.A., T.V. SAYGER et A.M. HORNE (1993). "Three types of family environment scale profiles: Functional, distressed, and abusive families », Journal of Family Violence, $8:$ 303-311.

Glasser, W. et W.T. POWERS (1981). Stations of mind: new directions for reality therapy. New York: Harper et Row.

Gottlieb, B.H. (1987). "Using social support to protect and promote health », Journal of Primary Prevention, $8: 49-70$.

GOUVERNEMENT DU QUÉBEC (1991). Un Québec fou de ses enfants : rapport du Groupe de travail pour les jeunes. Québec: Ministère de la Santé et des Services sociaux.

HART, S.N. (1988). "Psychological maltreatment: Emphasis on prevention", School Psychologist International, 9 : 243-255.

HART, S.N., R.B. Germain et M. BRASSARD (1987). "The challenge: To better understand psychological maltreatment of children and youth", dans M. Brassard, R. Germain et S. Hart (dir.), Psychological maltreatment of children and youth (p. 3-24). New York: Pergamon Press.

JOYAL, R. (1990). "La convention des Nations-Unies», Apprentissage et Socialisation, $13: 241-245$.

LAFORTUNE, D. et M.C. KeILEY (1989). «Prévention primaire des psychopathologies: appellation contrôlée», Santé mentale au Québec, vol. $14, \mathrm{n}^{\circ} 1$ : 54-68.

LUGTIG, D. et D. FUCHS (1992). Building of the strengths of local neighborhood social network ties for the prevention of child maltreatment. Unpublished manuscript.

MACMILLAN, H.L., J. MACMILLAN, D.R. OfFORD, L. GRIfFITH et A. MACMILLAN (1994). "Primary prevention of child physical abuse and neglect: A critical review. Part 1 ", Journal of Child Psychology and Psychiatry, vol. 35, $\mathrm{n}^{\circ} 5: 835-855$.

MAIN, M. et R. GOLDWYN (1984). "Predicting rejection on her infant from mother's representation of her own experience: Implications for the abused-abusing intergenerational cycle», Child Abuse and Neglect, $\mathrm{n}^{\circ} 8: 203-217$. 
MALO, C. (1995). Les mauvais traitements psychologiques envers I'enfant. Manuscrit non publié. Institut de recherche pour le développement social des jeunes, Montréal, $\mathrm{n}^{\circ} 10$.

MALO, C. (1991). Les programmes de prévention primaire et de promotion en santé mentale: de la conceptualisation à l'évaluation. Québec : Comité de la santé mentale du Québec.

National Research COUNCIL (1993). Understanding Child Abuse and Neglect. Washington, DC: National Academy Press.

NEY, P.G. (1987). "Does verbal abuse leave deaper scars: A study of Children and Parents», Canadian Journal of Psychiatry, $\mathrm{n}^{\circ} 34$ : 371-378.

OLDS, D.L. et H. KITZMAN (1990). "Can home visitation improve the health of women and children at environmental risk? », Pediatrics, $\mathrm{n}^{\circ} 86$ : 08116.

Olds, D.L., C.R. Henderson, R. Tatelbaum et R. Chamberlin (1986). «Improving the delivery of prenatal care outcomes of pregnancy: A randomized trial of nurse home visitation », Pediatrics, $\mathrm{n}^{\circ} 77: 16-28$.

Ouellet, F., D. DuRAND et MASSÉ (1989). Santé mentale au Québec.

Ouellet, F. et M. PAiEment (1993). "La démarche de concertation du Regroupement pour la qualité de vie (RVQ)», Journal sur le RVQ des jeunes de St-Laurent 1990-1993. MSSQ, p. 20-21.

PERREAUlt, R. (1995). "Stratégies de communication et promotion de la santé mentale», Prisme, vol. 5, no $1: 99-109$.

PRANSKY, J. (1991). Prevention. The critical need. Springfield: Burrell.

QUÉNIART, A. et F. FOURNIER (1995). Trajectoires de fragilisation du lieu père-enfant. Texte soumis pour les actes du Colloque "Enfances » de I'Association internationale des sociologues de langue française, 7.

ROOK, K.S. et D. DOOLEY (1985). "Applying social support research: Theoretical problems and future directions », Journal of Social Issues, $\mathrm{n}^{\circ} 41$ : 5-28.

SCHOOR, L. (1988). Within our reach: Breaking the cycle of disadvantage. New York: Anchor Books.

SCHORR, Lo B. (1988). Within our reach: Breaking the cycle of disadvantage and despair. New York: Doubleday.

StARR, R.H. et D.A. WOLFE (dir.) (1990). The effects of child abuse and neglect: Issues and research. New York: Guilford Press.

STRICKLAND, E.V. et K.C. CAMPBELL (1982). «End the other abuse: Damaged self-concepts », Childhood Education, n $8: 9-12$.

Tousignant, M., C. Chamberland, C. MAlO et L. Bozzini (1987). Utilisation de réseaux sociaux dans les interventions : état de la question et propositions d'action. Québec: Publications du Québec.

VEenstra, G.J. et C.G. SCOTT (1993). "A model for using time out as an intervention technique with families ", Journal of Family Violence, $\mathrm{n}^{\circ} 8: 71-89$.

Vissing, Y.M., M.A. Straus, R.J. Gelles et J.W. Harrop (1991). "Verbal aggression by parents and psychosocial problems of children », Child Abuse and Neglect, $\mathrm{n}^{\circ} 15: 223-238$.

WOLFE, D.A. (1985). "Child abusive-parents: An empirical review and analysis », Psychological Bulletin, $\mathrm{n}^{\circ} 97$ : 462-482. 